\title{
M87 AND THE X-RAY EMISSION FROM COMPACT OBJECTS
}

\author{
M. S. LONGAIR
}

Mullard Radio Astronomy Observatory, Cambridge, Great Britain

\section{Observations of $\mathbf{M 8 7}$ at $5 \mathrm{GHz}$}

(Abstract of a paper by I. Graham (1970))

M87 (Virgo A, 3C274) has been observed at $5 \mathrm{GHz}$ with the Cambridge One-Mile telescope, with a resolution of $6.5 \mathrm{arc} \mathrm{sec}$. These observations do not include the extensive halo but show that the central region of the source consists of at least three components. The central of the components is unresolved and appears to coincide with the optical nucleus, the outer pair extending from a point about 5 arc sec south of the optical nucleus. These components are unresolved perpendicular to their extension axes. The north preceding component is related to the optical jet but is more extended.

The physical conditions in the radio components and the optical jet are investigated in the light of these new observations. It is concluded that the two outer components probably consist of a number of small sub-components, each confined by its supersonic passage through the gas in the galaxy. It is shown that the nuclear component probably consists of two sub-components, the smaller of these producing the observed X-rays by the inverse Compton mechanism. The structure of the halo is used to derive information about the past activity of the source.

\section{The Diffusion of Relativistic Electrons from Infrared Sources and Their X-Ray Emission}

\section{(Abstract of a paper by A. S. Webster and M. S. Longair (1971))}

A model for X-ray sources in which relativistic electrons originate in compact nuclei which are also powerful sources of infrared emission is analyzed. The distortions of the injection electron spectrum due to inverse Compton losses and the spectrum of the resulting X-rays are determined for the case in which the propagation of the electrons from the nuclei can be described by a simple diffusion model. It is possible to explain the detailed features of the X-ray background spectrum in the range $0.25<\varepsilon_{x}<1000 \mathrm{keV}$ provided there is little dispersion in the properties of the sources. If Seyfert galaxies are the source of the X-ray background, their infrared spectra should have a maximum about $70 \mu \mathrm{m}$, in agreement with observation, and the diffusion coefficients should be $10^{29} \mathrm{~cm}^{2} \mathrm{~s}^{-1}$, similar to that of cosmic ray protons in the Galaxy.

\section{References}

Graham, I.: 1970, Monthly Notices Roy. Astron. Soc. 149, 319.

Webster, A. S., and Longair, M. S.: 1971, Monthly Notices Roy. Astron. Soc. 151, 261 


\section{Discussion}

Kellermann: It is difficult to understand the explanation of X-ray sources by inverse Compton scattering. The ratio of inverse Compton scattered X-rays to synchrotron radio emission depends only on radio brightness temperature. There are many compact radio sources of equally high brightness temperature as the compact component of M87 and with much greater radio synchrotron flux, that do not show X-ray emission.

Longair: Dr Kellermann's remark refers to models in which the infrared emission and the X-rays result from the same relativistic electrons. In our model these are decoupled. We postulate a compact source of infrared photons and relativistic electrons, both being generated by some unknown mechanism.

Hogg: The polarization structure of M87 at $11.1 \mathrm{~cm}$ has been measured by Dr Conway and myself, using the NRAO interferometer. The compact source associated with the nucleus is unpolarized, with an upper limit of $0.5 \%$. The two extended components are each polarized. The maximum polarization, $2.5 \%$, is found in the region of the optical jet.

Arp: An interesting detail is that the optical jet in M87 appears to emerge from the nucleus of M87 slightly offset to the north - only a few sec of arc but definitely noticeable on high resolution plates. The radio jets, on the other hand, now appear to center a few sec south of the nucleus.

A matter which now requires further optical investigation is what is the exact optical center of M87? The spot in the center of M87 (about 1.5 arc sec) which radiates strongly in [OII] emission appears to define the center on most photographic plates. It would be worthwhile now to determine independently the exact center of M87 from photographic plates in wavelengths which eliminate this $\lambda 3727 \AA$ emission. 